\title{
Vitamin A content in mature breast milk and its adequacy to the nutritional recommendations for infants
}

\author{
Natalia Matamoros, Biochemist ${ }^{a}$, Silvana Visentin, Biochemist ${ }^{a}$, Guillermina Ferrari, B.S. ${ }^{a}$, \\ Mariana Falivene, M.D.a, Victoria Fasano, M.D. ${ }^{a, b}$ and Horacio F. González, M.Da
}

\begin{abstract}
Objective. To determine vitamin A content in breast milk and evaluate whether it satisfies the recommendations for infants. Material and methods. Observational, prospective, cross sectional study. Milk samples were obtained between 30 and 90 days postpartum from mothers seen in public hospitals, and analyzed. Vitamin A concentration was determined by chromatography and its adequacy to the recommended dietary intake. The correlation between outcome measures was analyzed using Spearman's correlation coefficient.

Results. A total of 79 mothers participated. Vitamin A concentration in milk samples was $1.80 \mu \mathrm{mol} / \mathrm{L}$ (1.36-2.30) and the mean breastfeeding time was 57 days. No significant correlation between breastfeeding days and vitamin A content was observed. In $50 \%$ of the samples, vitamin A content did not satisfy the recommendations for infants.

Conclusion. Vitamin A content was not enough to satisfy the recommendations in half of the cases.
\end{abstract}

Key words: Vitamin A, breastfeeding, human milk.

http: / / dx.doi.org/10.5546/ aap.2018.eng.146

To cite: Matamoros N, Visentin S, Ferrari G, et al. Vitamin A content in mature breast milk and its adequacy to the nutritional recommendations for infants. Arch Argent Pediatr 2018;116(2):146-149.

a. Pediatric Research and Development Institute (Instituto de Desarrollo e Investigaciones Pediátricas, IDIP) "Prof. Dr. Fernando E. Viteri," Hospital de Niños "Sor María Ludovica," La Plata, Ministry of Health/ Scientific Research Commission (Comisión de Investigaciones Científicas, CIC), Province of Buenos Aires.

E-mail addres:

Horacio González, M.D.: horaciofgonzalez@gmail.com

Funding: This study was funded by the Institution itself (Pediatric Research and Development Institute [Instituto de Desarrollo e Investigaciones Pediátricas, IDIP] "Prof. Dr. Fernando E. Viteri").

Conflict of interest: None.

Received: 26-5-2017

Accepted: 9-12-2017

\section{INTRODUCTION}

The expression "vitamin A" includes retinol (preformed vitamin A) and the provitamin A carotenoids, which are dietary precursors of retinol. Vitamin is stored, mainly, in the liver. When needed, liver retinol is released into the blood bound to a retinol-binding protein to satisfy the body's physiological needs and, in breastfeeding mothers, to incorporate to breast milk. ${ }^{1,2}$ Vitamin A content in breast milk depends on the mother's nutritional status. ${ }^{3}$

In order to sustain an optimum visual, immune and cognitive development, fetuses, neonates and infants must receive enough vitamin A. ${ }^{4}$

Infants are born with a scarce vitamin A reserve in the liver due to the mother's homeostatic control, which regulates placental transfer of vitamin A to the fetus in order to prevent high concentrations of this vitamin to be passed to the child and harm him. Therefore, since birth to 6 months of age, breast milk is the only source of vitamin A to satisfy the child's needs and build his reserve. ${ }^{5}$

The purpose of this work was to determine vitamin A concentration in mature breast milk and assess its adequacy to the nutritional recommendations for infants.

\section{PATIENTS AND METHODS}

Study design and protocol: Observational, analytical, prospective, cross sectional study.

Inclusion criteria: Those mothers who had medical appointments or follow-up visits at the Observatory of the Pediatric Research and Development Institute (Instituto de Desarrollo e Investigaciones Pediátricas [IDIP]) "Prof. Dr. Fernando E. Viteri" at least one month after delivery, during the period 2012-2013, were invited to participate in the study. The milk samples were taken from mothers over 18 years old, who were exclusively breastfeeding, within 30 and 90 days postpartum, with a normal fetus and neonate history, and without vitamin treatment. The data used to characterize the study population were obtained from their medical records. 
The study protocol was approved by the IDIP's Institutional Research Protocol Review Committee, and the mothers who agreed to participate were requested to sign an informed consent. The study was carried out in accordance with the ethical standards set forth in the Universal Declaration of Human Rights of 1948, the Nuremberg Code and the Declaration of Helsinki of 1964, and successive revisions and amendments.

\section{Collection, transportation and storage of breast milk samples}

Breast milk samples were collected between 9:00 a.m. and 12:00 a.m. with an electric pump designed to extract breast milk (Medela), until the breast was completely emptied. Until their processing, the samples were stored under freezing conditions $\left(-70^{\circ} \mathrm{C}\right)$ and protected from light with an aluminum foil.

\section{Determination of vitamin A content in breast milk}

Vitamin A content in breast milk was determined using the procedure recommended by the Spanish Society of Clinical Biochemistry and Molecular Pathology (Sociedad Española de Bioquímica Clínica y Patología Molecular, SEQC) ${ }^{6}$ for the determination of total retinol in tissues.

The samples were analyzed by ultra-fast liquid chromatography (UFLC) with diode array detector, maximum absorption wavelength $(\lambda \max )$ of $325 \mathrm{~nm}$. The identification and quantification of retinol in the samples was done by comparison of retention times and areas of a standard of all-trans-retinol (SIGMA).

Data were expressed in $\mu \mathrm{mol} / \mathrm{L}$.

\section{Sample size}

The sample size was calculated in order to determine the mean concentration of vitamin $\mathrm{A}$ in mature milk with a 95\% confidence level and assuming an error of $0.10 \mu \mathrm{mol} / \mathrm{L}$ and a deviation of $0.47 \mu \mathrm{mol} / \mathrm{L} .7$

The final estimated size was of 78 milk samples.

\section{Analysis of results}

Adequacy to the dietary recommendations for infants was analyzed using the daily dietary reference intakes (DRI) for full-term infants between 0 and 6 months of age according to the Institute of Medicine of the US National Academy of Sciences. ${ }^{8}$
The statistical analysis was performed with the SPSS 18 statistical software for Windows. Quantitative outcome measures were expressed as the median with interquartile range (IQR), and Spearman's correlation coefficient was used to study the correlation between outcome measures.

\section{RESULTS}

Seventy nine samples of mature milk donated by mothers (mean age: 25 years; IQR: 20-30) who were exclusively breastfeeding were studied. The review of the mothers' medical records yielded the following results: $62.0 \%$ of them had a body mass index (BMI) above 25 , and $92.4 \%$ presented a body fat percentage of more than 30 . None of them was underweight. Gynecologically, $62 \%$ of them were multiparous. Table 1 shows the socio-economic characteristics of the breast milk donors.

Women had been breastfeeding for more than 30 days when the samples were taken (mature milk), with a median time of 57 (33-70) days.

Median vitamin A concentration in mature milk was $1.80 \mu \mathrm{mol} / \mathrm{L}(1.36-2.30)$.

Taking into account the DRI for infants between 0 and 6 months of age, and knowing that the estimated breast milk intake during the first six months of age was of $0.78 \mathrm{~L} /$ day, the analysis of adequacy showed that vitamin A content

TABLE 1. Characteristics of breast milk donors $(N=79)$

\begin{tabular}{lc}
\hline & $\mathbf{N}(\%)$ \\
\hline Occupation & $49(62.0)$ \\
Housewife & $3(3.8)$ \\
Student & $27(34.2)$ \\
Works outside the home & \\
Level of education & $6(7.6)$ \\
Incomplete primary education & $7(8.9)$ \\
Complete primary education & $20(25.3)$ \\
Incomplete secondary education & $25(31.6)$ \\
Complete secondary education & $11(13.9)$ \\
Incomplete tertiary/ univ. education & $10(12.7)$ \\
Complete tertiary/univ. education & \\
Family income & $4(5.1)$ \\
ARS 10-100 (USD 2-20) & $18(22.8)$ \\
ARS 100-1000 (USD 20-200) & $34(43.0)$ \\
ARS 1000-2000 (USD 200-400) & $23(29.1)$ \\
$>$ ARS 2000 (USD 400) & $40(50.6)$ \\
Unmet basic needs &
\end{tabular}

ARS: Argentine Pesos; USD: US Dollars.

(Exchange value 2012-2013). 
did not comply with such recommendations (400 $\mu \mathrm{g}$ of retinol/day) in $50 \%(\mathrm{n}=40)$ of the milk samples.

No significant correlation was found between breastfeeding time in days and vitamin A content in milk ( $\mathrm{p}=0.1407)$.

\section{DISCUSSION}

Median vitamin A concentration in breast mature milk was determined in this study, and it was found to be $1.80 \mu \mathrm{mol} / \mathrm{L}(1.36-2.30)$. Only $50 \%$ of the breast mature milks studied satisfied the dietary recommendations established by the DRI for infants between 0 and 6 months of age.

Newborn infants depend on breast milk to cover their needs and liver reserves. Studies show that retinol levels in breast milk usually decrease along the first year postpartum. This gradually reduces the mother-son retinol transfer through breastfeeding during the first year of life, since mean milk production also decreases between the first and the twelfth month in breastfeeding women. It has been assumed that this decreasing trend of retinol concentration in breast milk during postpartum is a sign of exhaustion of the mother's liver reserves, which is presumably related to vitamin A concentrations in breast milk. ${ }^{9}$ This reinforces the need for vitamin A supplementation during breastfeeding. ${ }^{10,11}$

Vitamin A transfer through breast milk during the first 6 months of life is 60 times higher than the transfer occurring through the placenta during the 9 months of pregnancy. If the mother's vitamin A nutritional status is deficient, children are susceptible to having vitamin A deficiency at the age of 6 months. Breastfeeding mothers and infants are considered population at risk of vitamin A deficiency. ${ }^{10,12,13}$

The results obtained in our study are consistent with those of two studies published by G. Souza et al., ${ }^{10,14}$ which assessed the content of vitamin A in breast mature milk of Brazilian mothers seen in public maternity centers and yielded values of $1.76 \pm 0.85$ and $1.87 \pm 0.81$ $\mu \mathrm{mol} / \mathrm{L}$, respectively. In those studies, $50.40 \%$ and $46.25 \%$ of breast milks, respectively, did not satisfy the DRI recommendations. Their results showed a high prevalence of a poor vitamin A nutritional status in mothers and children, consistent with the national prevalence reported for Brazilian children and women of childbearing potential.

When Oliveira and Marinho assessed fresh mature breast milk from 50 donors kept in a milk bank in Manaus, they also found that $58.40 \%$ of them did not satisfy the recommendations for infants. ${ }^{15}$

Based on our findings, which showed a high percentage of breast milk not complying with the recommendations regarding vitamin A content, studying the mother's nutritional status and intervening during pregnancy would improve breast milk quality and, hence, the nutritional status of infants exclusively breastfed.

A limitation of our work was that the mother and the baby's vitamin A nutritional status were not studied. Besides, the results are representative only of mature breast milk obtained from women seen in public hospitals in the city of La Plata.

\section{CONCLUSION}

The study proved that half of the breast milk samples obtained from the participants did not supply enough vitamin A to satisfy the US National Academy of Sciences' recommendations.

\section{REFERENCES}

1. Sherwin JC, Reacher MH, Dean $\mathrm{WH}$, et al. Epidemiology of vitamin A deficiency and xerophthalmia in at-risk populations. Trans R Soc Trop Med Hyg 2012;106(4):205-14.

2. Kam RK, Deng Y, Chen Y, et al. Retinoic acid synthesis and functions in early embryonic development. Cell Biosci 2012;2(1):11.

3. World Health Organization and Food and Agriculture Organization of the United Nations. Vitamin and mineral requirements in human nutrition. $2^{\text {nd }}$ ed. Hong kong: Joint FAO/WHO; 2004.

4. Fernandes TF, Andreto LM, Vieira CS, et al. Serum Retinol Concentrations in Mothers and Newborns at Delivery in a Public Maternity Hospital in Recife, Northeast Brazil. J Health Sci 2014;32(1):28-35.

5. Engle-Stone R, Haskell MJ, Nankap M, et al. Breast milk retinol and plasma retinol-binding protein concentrations provide similar estimates of vitamin A deficiency prevalence and identify similar risk groups among women in Cameroon but breast milk retinol underestimates the prevalence of deficiency among young children. J Nutr 2014;144(2):209-17.

6. Comisión de Vitaminas de la Sociedad Española de Química Clínica (SEQC). Procedimiento recomendado para la determinación de retinol en suero o plasma. Quím Clin 1998;17(1):38-42.

7. Matamoros N, Santandreu F, Disalvo L, et al. Contenido de vitamina A en leche materna madura después de la pasteurización: Requerimientos nutricionales del lactante. Rev Argent Salud Pública 2014;5(19):11-6.

8. Institute of Medicine. Dietary Reference Intakes: The Essential Guide to Nutrient Requirements. Washington, DC: The National Academies Press; 2006. [Accessed on: September 20th, 2017]. Available at: https://doi. org /10.17226/11537.

9. Fujita M, Shell-Duncan B, Ndemwa P, et al. Vitamin A dynamics in breastmilk and liver stores: a life history perspective. Am J Hum Biol 2011;23(5):664-73. 
10. SouzaG,DolinskyM,MatosA, etal.VitaminAconcentration in human milk and its relationship with liver reserve formation and compliance with the recommended daily intake of vitamin Ain pre-term and term infants in exclusive breastfeeding. Arch Gynecol Obstet 2015;291(2):319-25.

11. KingJC. The risk of maternal nutritional depletion and poor outcomes increases in early or closely spaced pregnancies. J Nutr 2003;133(5 Suppl 2):S1732-6.

12. Stoltzfus RL, Underwood BA. Breast milk vitamin A as an indicator to assess vitamin A status of women and infants. Bull World Health Organ 1995;73(5):703-11.
13. Elmadfa I, Meyer AL. Vitamins for the 1000 days: preparing for life. Int J Vitam Nutr Res 2012;82(5):342-7.

14. Souza G, Saunders C, Dolinsky M, et al. Vitamin A concentration in mature human milk. J Pediatr (Rio J) 2012;88(6):496-502.

15. Oliveira AM, Marinho HA. Determinaçaõ de vitamin Ano leite de măes doadoras do banco de leite humano (BLH) de Manaus/AM: efeito do processamento. Acta Amaz 2010;40(1):59-64.

\title{
Renal manifestations in children with Alagille syndrome
}

\author{
Diana Di Pinto, M.D. ${ }^{a}$ and Marta Adragna, M.D. ${ }^{a}$
}

\begin{abstract}
Introduction. Alagille syndrome (AS) is a cholestatic disease secondary to scarcity of interlobular bile ducts. It is associated with extrahepatic manifestations, and renal involvement is frequent.

Objectives. To describe the prevalence, type and outcome of renal pathology in children with AS.

Patients and Methods. The presence and outcome of renal pathology was retrospectively studied in 21 children who met AS criteria.

Results. Renal pathology was observed in 18 patients $(85.7 \%)$ :

(1) ultrasound variations in 7 patients (6 cases of bilateral renal dysplasia and 1 case of renal agenesis); (2) distal renal tubular acidosis in 2 patients; (3) a drop in glomerular filtration and / or proteinuria in 16 patients. The frequency of a drop in glomerular filtration was similar between patients with and without pathological kidney ultrasound findings.

Conclusions. Our study confirms a high prevalence of renal involvement, which enhances the importance of diagnosis and renal function follow-up in children with AS.

Key words: Alagille syndrome, kidney diseases

http:/ / dx.doi.org/10.5546/ aap.2018.eng.149
\end{abstract}

To cite: Di Pinto D, Adragna M. Renal abnormalities in children with Alagille syndrome. Arch Argent Pediatr 2018;116(2):149-153.

a. Department of Nephrology, Hospital Nacional de Pediatría "Prof. Dr. Juan P. Garrahan",

Autonomous City of Buenos Aires, Argentina.

E-mail address:

Diana Di Pinto, M.D.: dianadipinto@yahoo.com

Funding: None.

Conflict of interest: None.

Received: 7-13-2017

Accepted: 9-14-2017

\section{INTRODUCTION}

Alagille syndrome (AS) is a cholestatic disease secondary to scarcity of interlobular bile ducts, and associated to extrahepatic clinical manifestations. ${ }^{1}$ Its prevalence is estimated as 1 case per 70000 individuals and, in most cases, it is due to JAG1 gene mutations. ${ }^{2}$ Normally, it features five "classical" criteria: (1) intrahepatic cholestasis associated to ductopenia in liver biopsy (95\%); (2) heart malformations, typically, stenosis or hypoplasia of the pulmonary artery (92\%); (3) skeletal involvement, generally, butterfly vertebrae $(70 \%)$; (4) eye anomalies, often posterior embryotoxon (78\%); and (5) distinctive facial features $(91 \%)$, as wide forehead, ocular hypertelorism with sunken eyes, prominent ears, triangular face and straight nose. ${ }^{3-5}$ Three out of these five criteria are required for the diagnosis, or two if there is a family history. ${ }^{1}$

Renal involvement is variable (19-74\%), while renal dysplasia is the predominant manifestation (with or without cysts)..$^{1,5-7}$ Besides, distal renal tubular acidosis (dRTA), kidney cysts, urinary tract obstruction, renal agenesis, tubulointerstitial nephritis and vesicoureteral reflux can be observed. 2,4,7 Glomerular changes, like mesangiolipidosis and focal and segmental glomerulosclerosis have also been described, ${ }^{2,8}$ as well as arterial hypertension secondary to renal artery stenosis and other vascular involvements, like middle aortic syndrome, intra-abdominal vascular anomalies and central nervous system abnormalities ${ }^{3}$. Stroke was reported in up to $15 \%$ of cases. ${ }^{3}$

The purpose of this retrospective study was to describe the prevalence, type and outcome of renal 